\title{
Ground geomagnetic field and GIC response to March 17, 2015, storm
}

\author{
Olga V. Kozyreva ${ }^{1}$, Vyacheslav A. Pilipenko ${ }^{2 *}$, Vladimir B. Belakhovsky ${ }^{3}$ and Yaroslav A. Sakharov ${ }^{3}$
}

\begin{abstract}
The St. Patrick's Day geomagnetic storm on March 17, 2015, has been chosen by the space community for synergetic analysis to build a more comprehensive picture of the storm's origin and evolution. This storm had an unusually long $(\sim 17 \mathrm{~h})$ main phase. During this period, many substorm-like activations occurred. These activations resulted in bursts of geomagnetically induced currents (GICs) in power lines on the Kola peninsula. To examine the substorm activations in more detail, we apply various data processing techniques for the world-wide array of magnetometers: the virtual magnetograms, magnetic latitude-local time (MLT) snapshots, and magnetic keograms. These techniques are simple tools that are supplementary to more advanced facilities developed for the analysis of SuperDARN, IMAGE, and CARISMA arrays. We compare the global spatial localization and time evolution of the geomagnetic $X$-component disturbance and magnetic field variability measured by the Hilbert transform of time derivative $\mathrm{d} \mathbf{B} / \mathrm{d} t$. The latitude-MLT mapping of these magnitudes shows that very often a region with highest magnetic variability does not overlap with a substorm "epicenter" but is shifted to its poleward or equatorward boundaries. Highest variability of the geomagnetic field, and consequently intense GICs, are caused by medium-scale fast varying structures. There is no one-to-one correspondence between substorm intensity and GIC magnitude.
\end{abstract}

Keywords: Geomagnetic storm, Substorm, Geomagnetically induced currents, Virtual magnetometer

\section{Introduction}

The St. Patrick's Day geomagnetic storm on March 17, 2015 (day 076) is the largest in the 24th solar cycle (Ramsingh et al. 2015). This underpredicted storm is a peculiar magnetic event initiated by interaction between high-speed solar wind streams in a corotating interaction region (CIR) and two coronal mass ejections (CMEs) (Kamide and Kusano 2015). This storm has been chosen by the space community for scrutiny from different points of view. Such synergetic efforts may make it possible to build a more comprehensive picture of the storm's evolution. A fleet of space monitors crossed the bow shock, magnetopause, and plasmapause multiple times, and observed the magnetospheric compression that initiated the storm, and measured numerous ion injections (Goldstein et al. 2017). Satellite observations demonstrated that during the storm's main phase the

\footnotetext{
*Correspondence: space.soliton@gmail.com

${ }^{2}$ Geophysical Center, Moscow, Russia

Full list of author information is available at the end of the article
}

magnetopause moved inward to a location that is $\sim 5 R_{\mathrm{E}}$ closer to the Earth than expected based on pressure balance with the upstream solar wind (Le et al. 2016).

The Earth passed the portion of the magnetic cloud where the interplanetary magnetic field (IMF) was southward throughout the CME passage (Kataoka et al. 2015). The resultant southward IMF with exceptionally long duration was the main cause of this strong geomagnetic storm. The storm's main phase period lasted $\sim 17 \mathrm{~h}$, and the ring current index Dst reached $-228 \mathrm{nT}$. The storm's main and recovery phases were accompanied by a series of frequent and short-lived magnetic bays in the $X$ north-south (N-S) component. Not all of these magnetic bays can be associated with a substorm identified in auroral observations. Some bays were likely associated with the longitudinally localized auroral streamers and flow bursts (Lyons et al. 2016). Because of the localized nature of flow bursts, the standard auroral indices hardly can resolve them.

The storm of March 17, 2015, was very geoeffective. During this storm, auroral phenomena were observed 
even at very low latitudes. Van Allen Probes observations showed that relativistic electrons experienced rapid acceleration up to $\sim 7 \mathrm{MeV}$ within two days after the initial substantial dropout ( $\mathrm{Li}$ et al. 2016). A significant factor of the storm's geoeffectiveness is the magnetic field variability, characterized by time derivative $\mathrm{dB} / \mathrm{d} t$ (Boteler et al. 1998). The $\mathrm{dB} / \mathrm{d} t$ effect of magnetic spikes causes geomagnetically induced currents (GICs) that may have damaging effects on ground-based technology systems (Lanzerotti 2001). GICs are produced by fast medium-scale variations of the geomagnetic field rather than much more intense large-scale current systems (Viljanen 1997). Therefore, the examination of fine features of the geomagnetic field variability is very important not only for understanding the fundamentals of magnetosphere-ionosphere coupling, but also for predicting GIC intensity. A set of efficient data analysis tools is necessary to reveal fine features of geomagnetic field variations.

Using the satellite terminology, nowadays one can see in geomagnetism an ever-growing transfer from analysis of L2 data (calibrated data from a single site) to L3 dataderived global and regional models. Here we present some processing techniques for the analysis of data from a world-wide network of geomagnetic observatories. These simple techniques are supplementary tools to the advanced facilities developed for the analysis of SuperDARN, IMAGE, and CARISMA arrays. We apply relatively new processing techniques for the analysis of data from a world-wide network of geomagnetic observatories during the March 17, 2015, storm. Using this storm as an example, we examine the spatial/temporal correspondence between multiple substorm-like intensifications and magnetic field variability during the storm's main phase. Implications of magnetic field variability analysis for bursts of GIC recorded in power transmission lines are discussed.

\section{Database used}

Interplanetary parameters and geomagnetic indices

The 1-min solar wind and IMF parameters from ACE/ WIND satellites are provided by the NASA OMNI-2 database, albeit with some data gaps. The AE index is a measure of the ionospheric electrojet global intensity in the auroral zone. A similar auroral SME index is produced within the frameworks of the SuperMAG project, but from a much larger number of stations $(\sim 110)$ (Newell and Gjerloev 2011). The oval boundaries can be identified by the OVATION model, based on low-orbit DMSP particle measurements.

The polar cap PC index is derived from magnetic data of stations located in the northern (PCN) and southern (PCS) near-pole regions, and commonly considered as a proxy for energy that enters the magnetosphere during solar winds-magnetosphere coupling (Stauning 2018).

\section{Global magnetometer array}

The world-wide array ( 140 stations) of fluxgate magnetometers comprises CARISMA, INTERMAGNET, MACCS, International Monitor for Auroral Geomagnetic Effects (IMAGE), Greenland Coastal, GIMA, MAGDAS, and Russian Arctic networks in the Northern hemisphere. This database was supplemented with data from stations in the Southern hemisphere (Antarctica). Codes, geographic and geomagnetic coordinates of these stations can be found on relevant websites given below.

We have worked with two types of input data: (A) the data from all available magnetometer arrays (INTERMAGNET, IMAGE, Greenland, etc.) and single stations are compiled into a unified format and 1-min sampling rate, and rotated, if necessary, into the geographical coordinate system $(X, Y, Z)$. The baseline-the average daily value or the value at the day onset, is subtracted; (B) for a case study, it is possible to download data from the SuperMAG portal in a unified format in local geomagnetic coordinate system and baseline subtracted (Gjerloev 2012). The comparison of resulting plots produced from types (A) and (B) data with different detrending procedures provides similar results. Also, plots for magnetic field components either in the corrected geomagnetic or the geographic coordinates look nearly the same.

\section{GIC stations}

The Polar Geophysical Institute (Apatity) maintains a network of stations for recording GIC in electric power transmission lines (Sakharov et al. 2009; Viljanen 2011). This network extends in the latitudinal direction on the Kola peninsula and in Karelia. Each station records a quasi-DC current in the dead-grounded neutral of a transformer with a $10 \mathrm{~s}$ sampling rate. In this study we use data from stations Vykhodnoj (VYH) at a $330-\mathrm{kV}$ power line and Revda (REV) at a $110-\mathrm{kV}$ power line. The coordinates of the GIC-recording stations and of the near-by magnetic observatories Ivalo (IVA) and Sodankyla (SOD) are given in Table 1.

Table 1 Magnetometer and GIC stations

\begin{tabular}{lllllll}
\hline Station & Code & & \multicolumn{2}{c}{ Geographic } & & \multicolumn{2}{c}{ Geomagnetic } \\
\cline { 7 - 8 } & & Lat. & Long. & & Lat. & Long. \\
\hline Ivalo & IVA & 68.70 & 27.30 & & 65.0 & 109.8 \\
Sodankyla & SOD & 67.37 & 26.63 & & 63.8 & 107.7 \\
Vykhodnoj & VYH & 68.83 & 33.08 & & 65.0 & 114.1 \\
Revda & RVD & 67.77 & 34.99 & & 63.8 & 114.9 \\
\hline
\end{tabular}




\section{Data analysis tools}

\section{Virtual magnetograms}

A serious drawback of the analysis of ground-based magnetograms is the inevitable variation of the magnetic response due to continual changes in the station location in magnetic local time. An ideal, but impossible solution of this difficulty that will help to discriminate temporal and spatial variations would be the deployment of a "stationary" in situ observatory with a fixed position in the solar-magnetospheric coordinate system. However, the desired result can be obtained with the proposed technique of "virtual magnetogram" (VM). VM may be used as an additional tool for monitoring the response of the geomagnetic field to solar wind and IMF forcing. This technique has been implemented for the noon cusp, geomagnetic latitude $\Phi= \pm\left(75^{\circ} \pm 5^{\circ}\right)$, and midnight auroral, $\Phi= \pm\left(65^{\circ} \pm 5^{\circ}\right)$, regions. VM for a fixed reference system was reconstructed by $2 \mathrm{D}$ fitting and interpolation of 1-min magnetograms from magnetic stations nearest to a "virtual magnetometer". Plots of VMs show as well simultaneous interplanetary parameters: the solar wind density $N p$, interplanetary electric field $E y=-V^{*} B z$ (where $V$ is the solar wind velocity, and $B z$ is the northsouth IMF component), and the geomagnetic ring current SYM-H, auroral $\mathrm{AE}$, and polar cap $\mathrm{PCN} / \mathrm{PCS}$ indices. The spatial coverage of magnetometers is rather poor in some regions, so reliability of VM may vary depending on the current location of the virtual magnetometer. The provisional database of VMs for recent years is freely available at http://vm.gcras.ru.

\section{Latitude-MLT snapshots}

The global structure of magnetic activity can be characterized by maps (snapshots) in the Geomagnetic Latitude $(\Phi)$-Magnetic Local Time (MLT) coordinates of global distribution of the disturbance amplitude. To estimate magnetic disturbance magnitude $\Delta \mathbf{B}$, the value of the magnetic field before the storm onset is used as a reference level.

An instant amplitude of disturbance is estimated with the use of the analytical signal presentation based on the Hilbert transform. At each site, a $\pi / 2$-phase-shifted time series $B_{i}(t)$ is found for a detrended time series $B_{r}(t)$ with the use of Hilbert transform. An instant amplitude (envelope) $|B(t)|=\sqrt{B_{r}^{2}+B_{i}^{2}}$ is calculated for each horizontal magnetic component $(X, Y)$ for a resulting analytical signal $B=B_{r}+B_{i}$.

The global structure of the disturbance is characterized by a $2 \mathrm{D}$ map constructed by interpolating the amplitude envelopes at an array of stations for a selected time moment. Upon map construction, we grid the data transferring them from an irregular 2D network of sites to a regular net. This non-trivial problem has been addressed by SuperMAG community (Gjerloev 2012). As a first step in resolving this problem we have applied the simple "inverse distance" algorithm, which has been used for the construction of both VMs and 2D maps. For each UT moment, 5 nearest stations to a chosen location in a given range of geomagnetic latitudes are found. Then a value of geomagnetic disturbance at this location is calculated as the weighted average of the measured disturbances from those 5 stations as follows

$$
A_{0}=\frac{\sum_{i=1}^{5} A_{i} d_{i}}{\sum_{i=1}^{3}\left|d_{i}\right|} \quad d_{i}^{-1}=\sqrt{\left(\mathrm{MLT}_{i}-\mathrm{MLT}_{0}\right)^{2}+\left(\Phi_{i}-\Phi_{0}\right)^{2}}
$$

Here $A_{i}=\{X, Y\}$ is the disturbance amplitude at site $(i), d_{i}$ is the weight coefficient depending on the MLT/latitude difference between a given location and site $(i)$. This algorithm returns good results for close stations, but it provides a reduced value for very large distances between the stations and virtual/grid point. The experiments with other gridding techniques are under way.

Magnitude $\Delta X$ of the $X$-component magnetic bay is used to characterize the global structure of magnetic activity and the ionospheric electrojet intensity. To quantify the magnitude of the magnetic field variability, time derivatives $\mathrm{d} X / \mathrm{d} t$ and $\mathrm{d} Y / \mathrm{d} t$ are calculated. The earlier examinations of $\Delta \mathbf{B}$ and $\mathrm{d} \mathbf{B} / \mathrm{d} t$ spatial structures revealed the occurrence of localized (scales about $10^{3} \mathrm{~km}$ and less) structures embedded in the main electrojet (Viljanen 1997). Current amplitudes in such structures can be small, so they hardly can be discriminated from global $\Delta \mathbf{B}$ pattern. However, the temporal changes associated with these medium-scale structures are fast, so they are clearly seen in the $\mathrm{d} \mathbf{B} / \mathrm{d} t$ distribution. In magnetograms, this feature is observed during disturbed periods as a large $\Delta X \gg \Delta Y$, but about equal time derivatives $\mathrm{d} X / \mathrm{d} t$ and $\mathrm{d} Y / \mathrm{d} t$ (Viljanen et al. 2001). Therefore, for the analysis we use the magnitude of the total time derivative of the horizontal magnetic component $|\mathrm{d} \mathbf{B} / \mathrm{d} t|=\sqrt{(\mathrm{d} X / \mathrm{d} t)^{2}+(\mathrm{d} Y / \mathrm{d} t)^{2}}$.

Envelopes of $\Delta X(t)$ and $|\mathrm{d} \mathbf{B} / \mathrm{d} t|$ are determined with the method of analytical signal. The global structure of the activity is characterized by $2 \mathrm{D} \Phi$-MLT maps of the amplitude envelope of $\Delta X$ and $|\mathrm{d} \mathbf{B} / \mathrm{d} t|$. Whenever possible, these $2 \mathrm{D}$ plots are augmented with a current position of the auroral oval. The oval boundaries were identified by the OVATION model, based on low-orbit DMSP particle measurements.

\section{Magnetic keogram}

The "magnetic keogram" shows the time evolution of the latitudinal structure of magnetic disturbance, like optical 
keograms used in analysis of a meridional-scanning photometer. For that, an instant amplitude of magnetic disturbance $\Delta X(t)$ from all the stations of a latitudinal array (e.g., IMAGE) has been calculated with the Hilbert transform. Then, a color-coded latitudinal distribution of $\Delta X$ is plotted in a running time window (e.g., $20 \mathrm{~min}$ ). Such a plot is, in effect, a simplified version of the equivalent ionospheric 1D current plot, developed in the Finnish Meteorological Institute-FMI (http://space.fmi.fi/image /). The FMI color plots show the time evolution of the ionospheric E-W currents along a N-S profile.

A similar technique has been applied to construct "keograms" of the magnetic field variability $|\mathrm{d} \mathbf{B} / \mathrm{d} t|$. A keogram shows not only the magnitude of the $|d \mathbf{B} / \mathrm{d} t|$ burst, but its latitudinal scale and peak location as well. This information is critically important to estimate a GIC magnitude in a specific power transmission line.

\section{Interplanetary and magnetospheric conditions during the storm}

The interplanetary shock preceding the CME arrival is detected by the WIND/ACE and GEOTAIL spacecraft at 0445 UT, March 17 (Fig. 1). During the shock, the IMF experiences strong variations, and IMF $\mathrm{Bz}$ becomes more strongly positive. A rapid and intense increase in the solar wind plasma density $N p$ up to $\sim 35 \mathrm{~cm}^{-3}$, and the dynamic pressure $P$ up to $\sim 15 \mathrm{nPa}$ occurs at $\sim 05$ UT. The density increase is accompanied by velocity $V$ increase from $\sim 400$ to $\sim 500 \mathrm{~km} / \mathrm{s}$. The shock hits the magnetosphere at $\sim 0446 \mathrm{UT}$, inducing the impulsive disturbance (sudden commencement) and transient quasi-periodic Pc5 pulsations. The storm's main phase follows the IMF $B z$ southward turn at $\sim 06 \mathrm{UT}$. The IMF $B z$ then fluctuates between north and south before it stays strongly southward around $-20 \mathrm{nT}$ for a prolonged period, which causes a very gradual storm growth. The storm's main phase lasts $\sim 17 \mathrm{~h}$ and reaches a maximum (SYM-H $230 \mathrm{nT}$ ) at the end of March 17. The AE index shows a series of intense enhancements, above $2000 \mathrm{nT}$, associated with more or less isolated substorms during the long main phase. Even during the recovery phase under a quasi-steady solar wind flow and IMF $B z$ oscillating around zero, a series of substantial AE index spikes, above $1000 \mathrm{nT}$, occurs. Most of sudden AE increases are accompanied by bursts of GIC in power transmission lines (Fig. 1, bottom panel).

Here we demonstrate how the techniques described above work for the case of the March 17, 2015, storm. A concise view of the geomagnetic response to the disturbed space weather during the storm's main phase can be seen in the noon and midnight VMs for the northern and southern hemispheres (Fig. 2, two bottom panels). Under positive Ey many substorm-like enhancements of the $\mathrm{AE}$ index took place. We consider six $\mathrm{AE}$ spikes during the main phase, possibly corresponding to substorms, marked in Fig. 2 with numbers: \#1) elevated AE from 06 UT till $11 \mathrm{UT}$, \#2) peak of AE $2800 \mathrm{nT}$ at 1341 UT, \#3) short burst of AE up to $1600 \mathrm{nT}$ with peak at $1650 \mathrm{UT}$, \#4) elevated burst-like AE up to $1650 \mathrm{nT}$ during 1720-1840 UT, \#5) elevated AE up to $1600 \mathrm{nT}$ during 20-22 UT, and \#6) peak AE $2300 \mathrm{nT}$ at 2344 UT.

Magnetic bays at VM correspond to the occurrence of all 6 auroral activations (Fig. 2, bottom panels), though the magnitudes of the magnetic bays are not linearly proportional to the pertinent AE values. Period \#1 (0611 UT) was thoroughly examined by Lyons et al. (2016) using optical, radar, and magnetometer data from the North American sector. Irregular disturbances during this time interval were interpreted to result from rapid poleward/equatorward expansions and auroral streamers of various intensities. VM also demonstrates the fine structure of this disturbed period and indicates onsets of each magnetic bay more clearly than the AE index. In particular, VM shows the occurrence of several substorm-like activations (especially evident from VM for the Southern hemisphere), which are hard to distinguish from AE index data only. Besides that, VMs provide qualitative visual information about the day-night coupling and $\mathrm{N}-\mathrm{S}$ asymmetry. For example, during activation \#1, VMs give a possibility to discriminate a dayside response to $E y$ increase, and a nightside substorm. At the same time, VM characterizes the nighttime activity throughout the day better than AE index or the IMAGE electrojet index IE (not shown). The SuperMAG SME index for this event has an off-set about $400 \mathrm{nT}$ with the standard $\mathrm{AE}$ index (Fig. 1).

\section{Global distribution of the ionospheric electrojet intensity and geomagnetic field variability}

During the very long main phase of the storm, a few substorms and substorm-like activations with intensity $\mathrm{AE}>1000 \mathrm{nT}$ occur, often overlapping. Here we present global maps of the spatial structure of the disturbed magnetic field constructed from world-wide data during the most prominent geomagnetic intensifications:

\section{Substorm \#2 (1333 UT)}

The substorm originates in the post-midnight sector (Fig. 3, upper panel). The maximum intensity of $\Delta X$ disturbance (and correspondingly, the westward auroral electrojet) is near the equatorward boundary of the OVATION auroral oval, at $\Phi \sim 62^{\circ}$.

However, maximum variability of the geomagnetic field $|\mathrm{d} \mathbf{B} / \mathrm{d} t|$ during this period is observed near the poleward boundary of the auroral oval, $\sim 72^{\circ}$ (Fig. 3, bottom panel). 


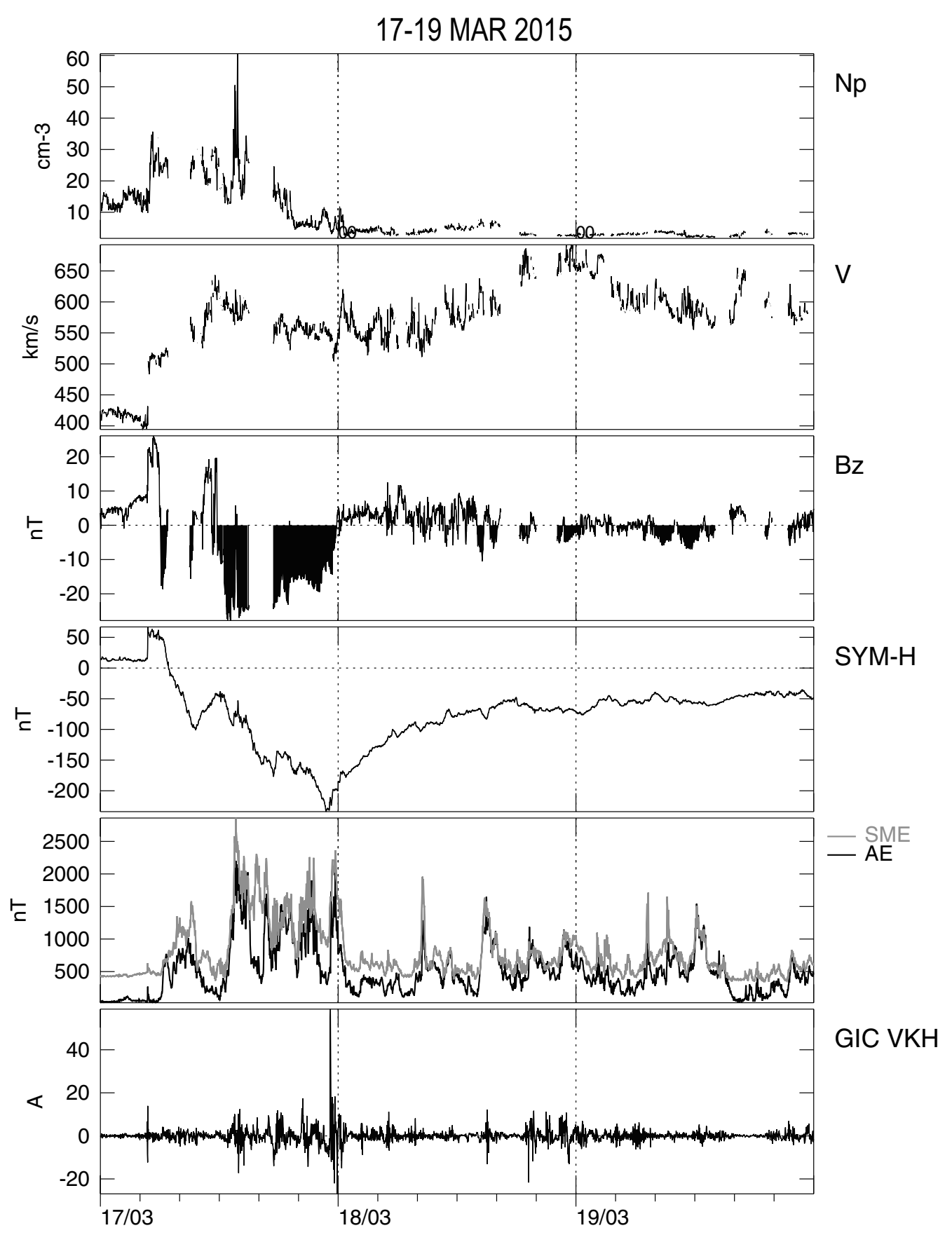

Fig. 1 Space weather parameters during the March 17-19, 2015, storm: the solar wind density Np and velocity V, IMF BZ, SYM-H ring Current index, $\mathrm{AE}$ and SME auroral indices, and GIC intensity (in Amperes) at station VYH

\section{Substorm \#4 (1748 UT UT)}

This substorm originates at $\Phi \sim 56^{\circ}$ in the post-midnight hours ( 02 MLT). Upon its evolution, the substorm epicenter expands westward to the late evening sector $\sim 20-22$ MLT. As a result, maximum magnetic disturbance $\Delta X$ is observed in a latitudinally localized region at $\Phi \sim 60^{\circ}-65^{\circ}$ from the pre-midnight hours to post-midnight hours at $\Phi \sim 56^{\circ}$ (Fig. 4, upper panel). The OVATION model data are not available for this period.

Maximum variability of the geomagnetic field $|\mathrm{d} \mathbf{B} / \mathrm{d} t|$ is also observed in the pre-midnight sector ( $18 \mathrm{LT})$, but at higher latitude $\Phi \sim 66^{\circ}$ (Fig. 4, bottom panel). 

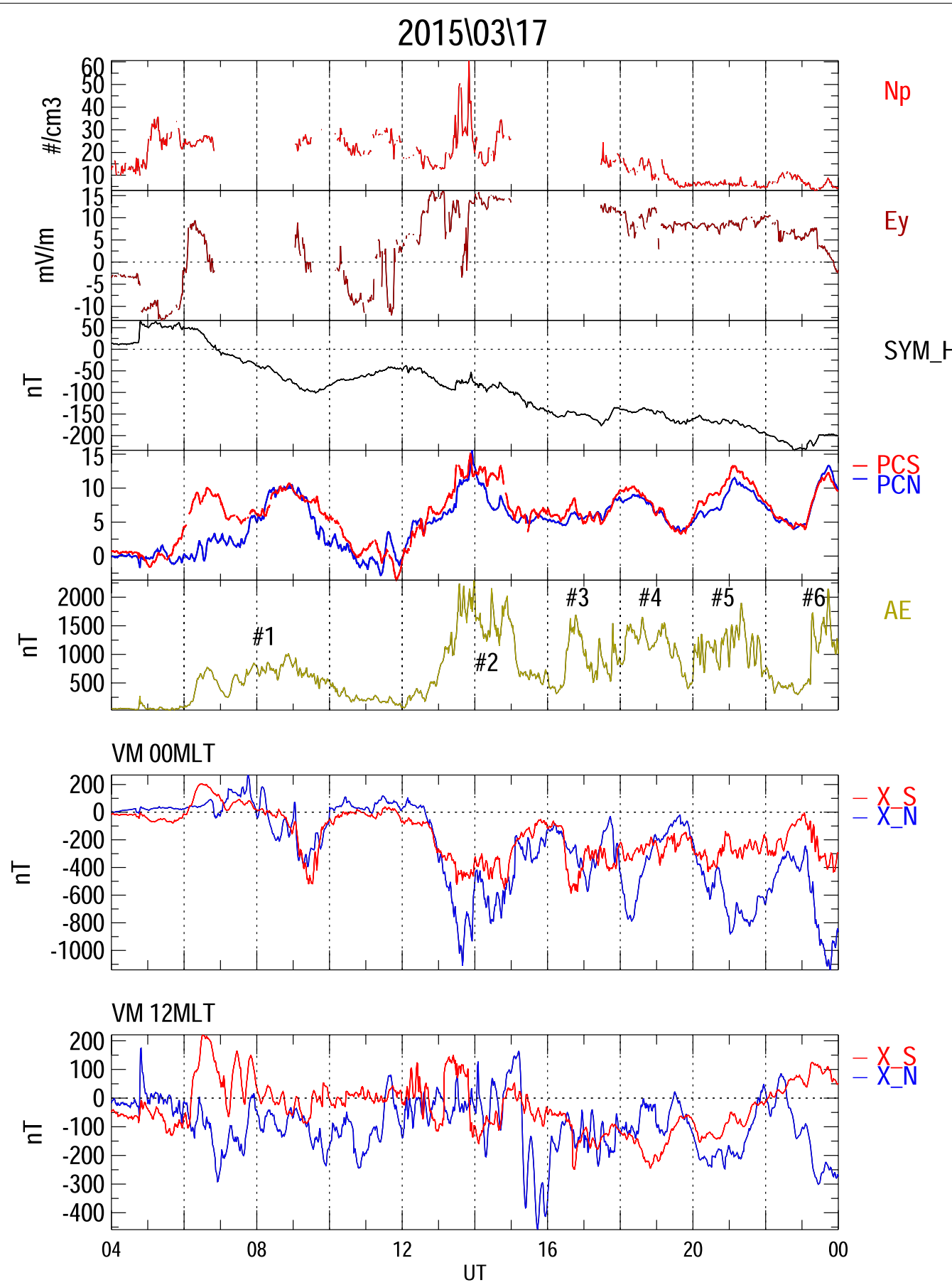

Fig. 2 The space weather parameters during the storm main phase 04-24 UT, March 17, 2015: the solar wind density Np and electric field Ey, SYM-H index, PCN/PCS indices, and AE index. Two bottom panels show the midnight (MLT $=0)$ and noon $(M L T=12)$ virtual magnetograms (X-component) of geomagnetic response for the Northern (blue line) and Southern (red line) hemispheres. Substorm activations are marked by numbers (1-6)

Substorm \#6 (2316 UT)

Substorm intensification is observed in the early morning sector ( 02-04 LT) with maximum intensity at $\Phi \sim 60^{\circ}$
(Fig. 5, upper panel). The spatial peak of variability $\mid \mathrm{d} \mathbf{B} /$ $\mathrm{d} t \mid$ is in the same sector, but at a higher latitude, $\Phi \sim 66^{\circ}$.

Thus, the global $\Phi$-MLT snapshots of $\Delta X$ and $|\mathrm{d} \mathbf{B} / \mathrm{d} t|$ reveal that during considered substorm intensifications 

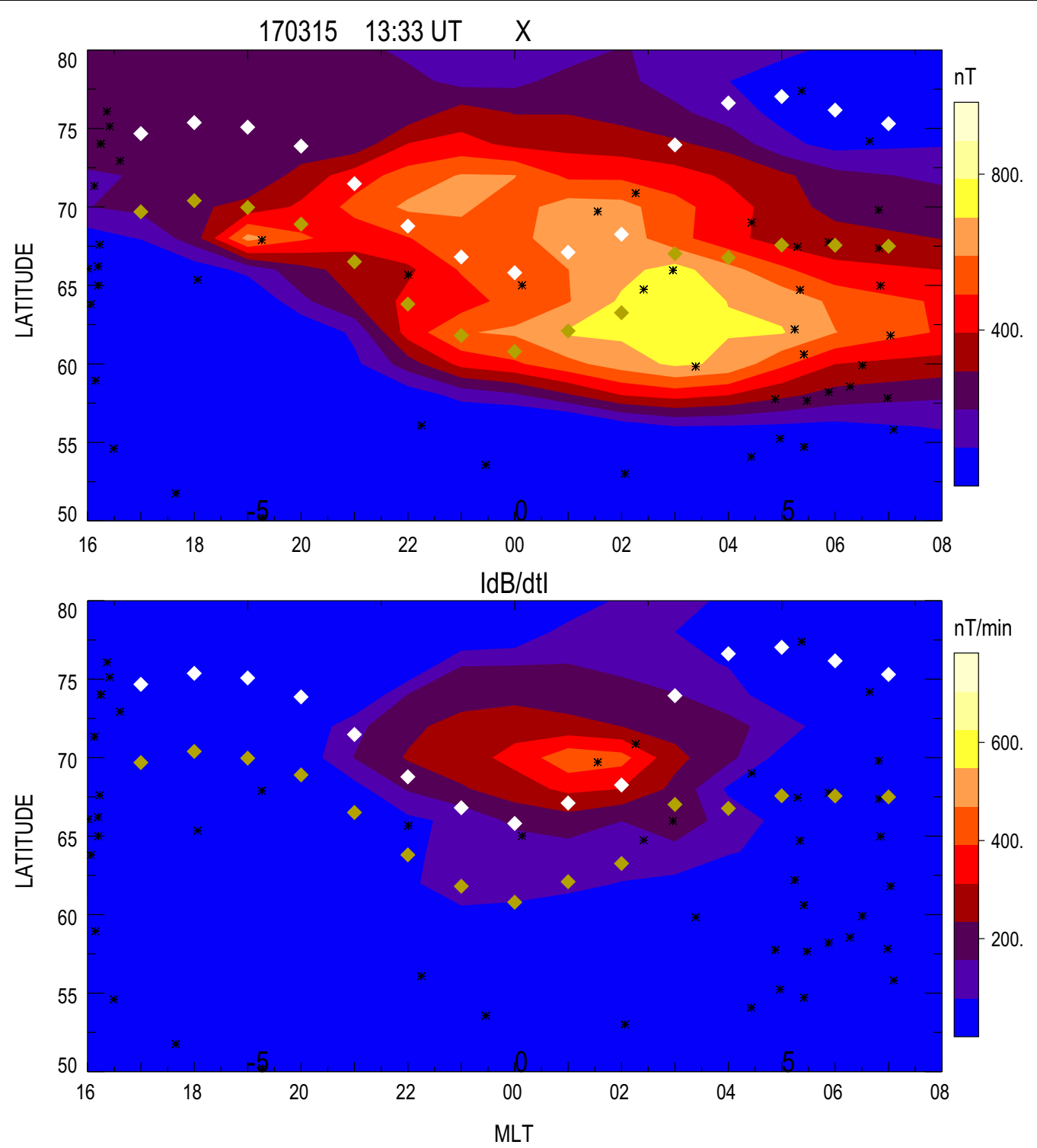

Fig. 3 World-wide distribution of magnetic disturbance magnitude for the time moment 1333 UT (substorm \#2) on March 17, 2015, as characterized by the global $\Phi$-LT maps of $\Delta X$ variations (upper panel), and magnetic variability $|\mathrm{d} \mathbf{B} / \mathrm{d} t|$ (bottom panel). Small black crosses denote the station location. Black and yellow diamonds denote the poleward and equatorward boundaries of the auroral oval, given by the OVATION model

maxima of $|\mathrm{d} \mathbf{B} / \mathrm{d} t|$ are located not in the center of the auroral electrojet, but around its boundaries.

\section{Dynamics of latitudinal distribution of magnetic field disturbance and variability}

A magnetic keogram of $\Delta X$ along the IMAGE latitudinal profile (geomagnetic longitude $\Lambda \sim 110^{\circ}$ ) shows the time evolution of the intensity and instant localization of the substorm "epicenter", that is, the intensity of the magnetic bay measured by the $X$-component (Fig. 6). The magnetic keogram clearly indicates the origin and evolution of all intensifications evident from the $\mathrm{AE}$ index and $V M$, except for substorm \#3 which takes place far from the IMAGE array. During substorm \#1, weak intensifications are observed at high latitudes, around $70^{\circ}-75^{\circ}$. During substorm \#2, an intense negative bay (corresponding to westward electrojet) develops at $\Phi>69^{\circ}$, and a positive bay (eastward electrojet) occurs in the latitudinal range $58^{\circ}-62^{\circ}$. The subsequent substorms \#4-6 develop at lower latitudes. 

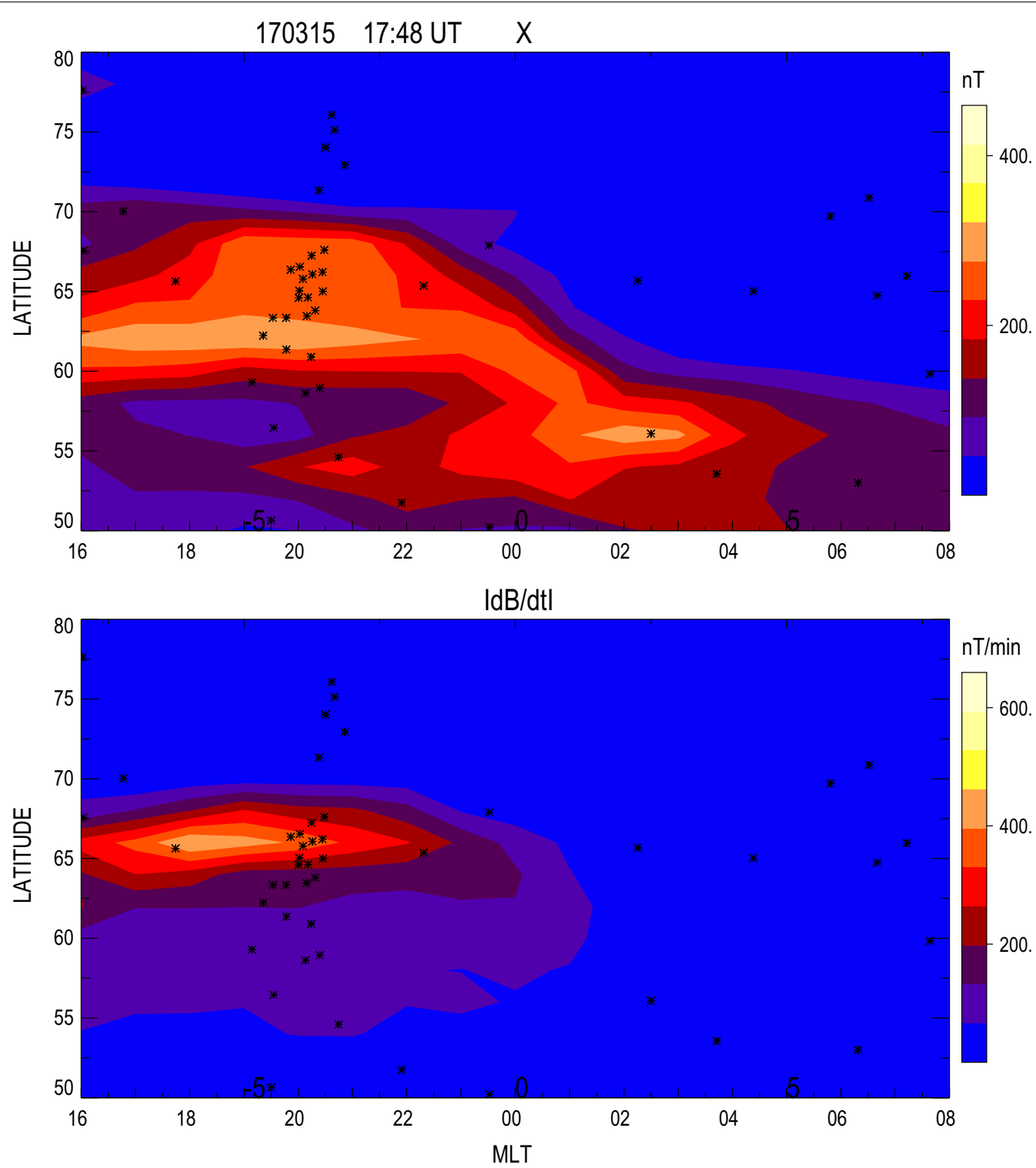

Fig. 4 Global $\Phi$-LT maps for the time moment 1748 UT (substorm \#4) of the $\Delta X$ variations (upper panel) and magnetic variability |dB/dt| (bottom panel). Small black crosses denote the station location. No OVATION model data are available for this period

The keogram of magnetic field variability $|\mathrm{d} X / \mathrm{d} t|$, also along the IMAGE profile, shows when and where bursts of $|\mathrm{d} X / \mathrm{d} t|$ take place (Fig. 7). The burst at $~ 0446 \mathrm{UT}$ is caused by sudden commencement. The largest $|\mathrm{d} X / \mathrm{d} t|$ enhancements are recorded during substorms \#2 and \#6 around $63^{\circ}-65^{\circ}$ and $58^{\circ}-60^{\circ}$. The latitudinal scale of the region with elevated $|\mathrm{d} X / \mathrm{d} t|$ magnitude is $\Delta \Phi \sim 2^{\circ}-4^{\circ}$. This information is critically important for the problem of GIC occurrence in a specific power transmission line. Significant GIC would be observed only when a region with high field variability overlaps with a power line under question.
Comparison of Figs. 6 and 7 indicates that largest intensities of $|\mathrm{d} \mathbf{B} / \mathrm{d} t|$ are observed not in the epicenters of the magnetic disturbances, but at their poleward or equatorward boundaries. For example, during the largest substorm \#2, the maxima of the westward and eastward electrojets are at $\Phi \sim 71^{\circ}$ and $\Phi \sim 61^{\circ}$, whereas maximum variability is around $63^{\circ}-65^{\circ}$. Similarly, during substorm \#6 maximum disturbance $\Delta X$ is between $62^{\circ}-65^{\circ}$, whereas maximum of $|\mathrm{d} X / \mathrm{d} t|$ is at $\Phi \sim 58^{\circ}$. 

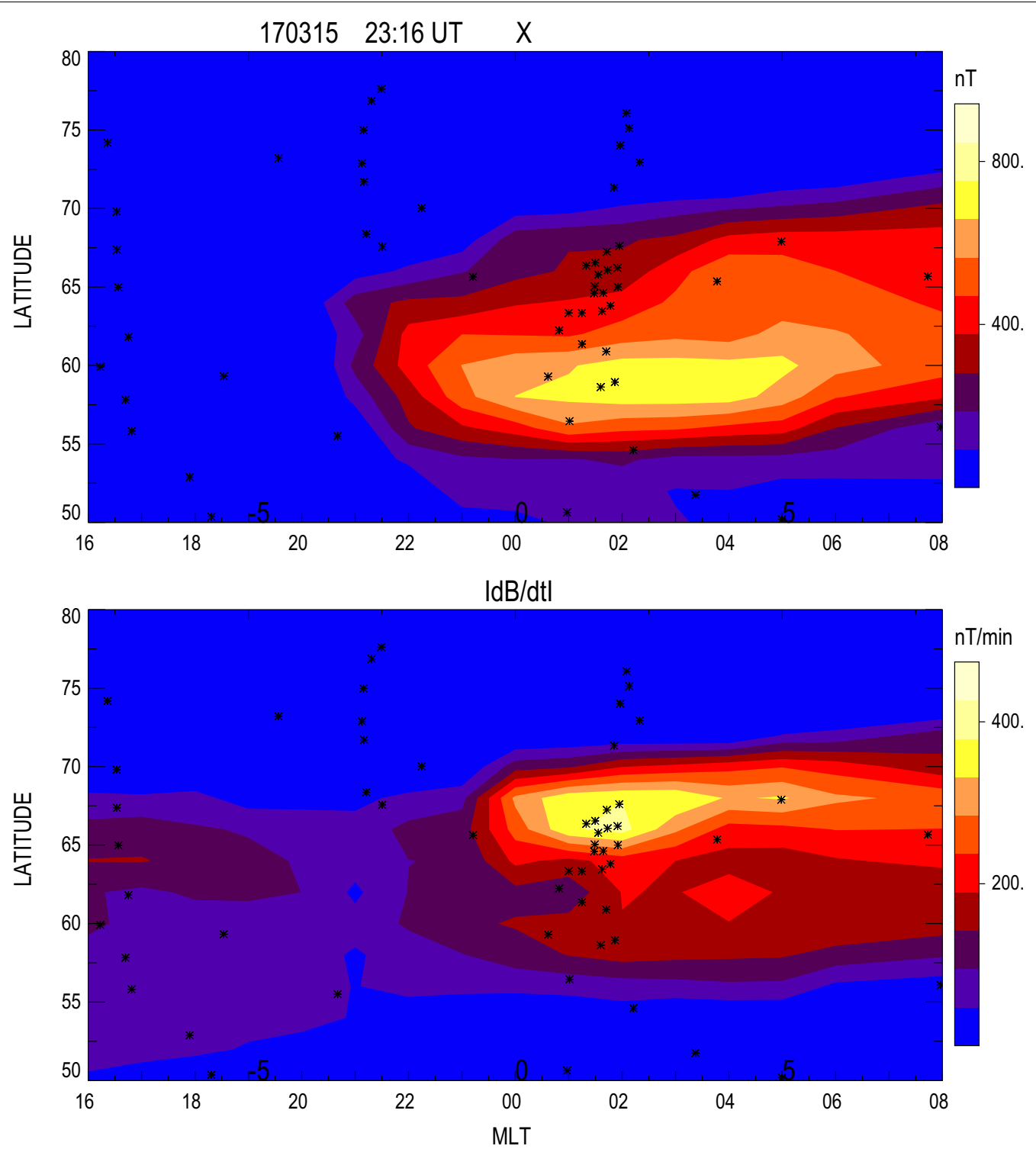

Fig. 5 Global $\Phi$-LT maps for the time moment 2316 UT (substorm \#6) of $\Delta X$ variations (upper panel), and magnetic variability |dB/dt| (bottom panel). No OVATION model data are available for this period

\section{Magnetic field variability and GIC}

The GIC intensity is determined by the time variations of the geomagnetic field $\mathrm{d} \mathbf{B} / \mathrm{d} t$. We examine the relative contribution of various components of geomagnetic disturbances to the generation of GIC using data from a GIC-recording system and near-by magnetometers from the IMAGE array. An approximate rule for interpreting vector distributions is that $\mathrm{dB} / \mathrm{d} t$ rotated $90^{\circ}$ anticlockwise is parallel to the electric field $\mathbf{E}$ at the Earth's surface (strictly true if the disturbance field is a plane wave and the Earth's conductivity is laterally homogeneous). The distribution patterns of $\mathrm{d} \mathbf{B} / \mathrm{d} t$ cannot be explained by a simple line current model of the ionospheric currents, because rapidly changing medium-scale field-aligned currents must play an important role.

GICs in the electric power transmission line at stations VYH and KND on the Kola peninsula are shown in Fig. 8d, g. These GICs are compared with magnetic field variations at the nearest magnetometers IVA and SOD. This comparison shows that every substorm, as measured by the $\mathrm{AE}$ index (upper panel), results in an enhancement of GIC. However, the GIC magnitudes are different: slowly growing intensifications starting on $\sim 08 \mathrm{UT}$ and $\sim 0930$ UT produce weak GIC enhancements as 


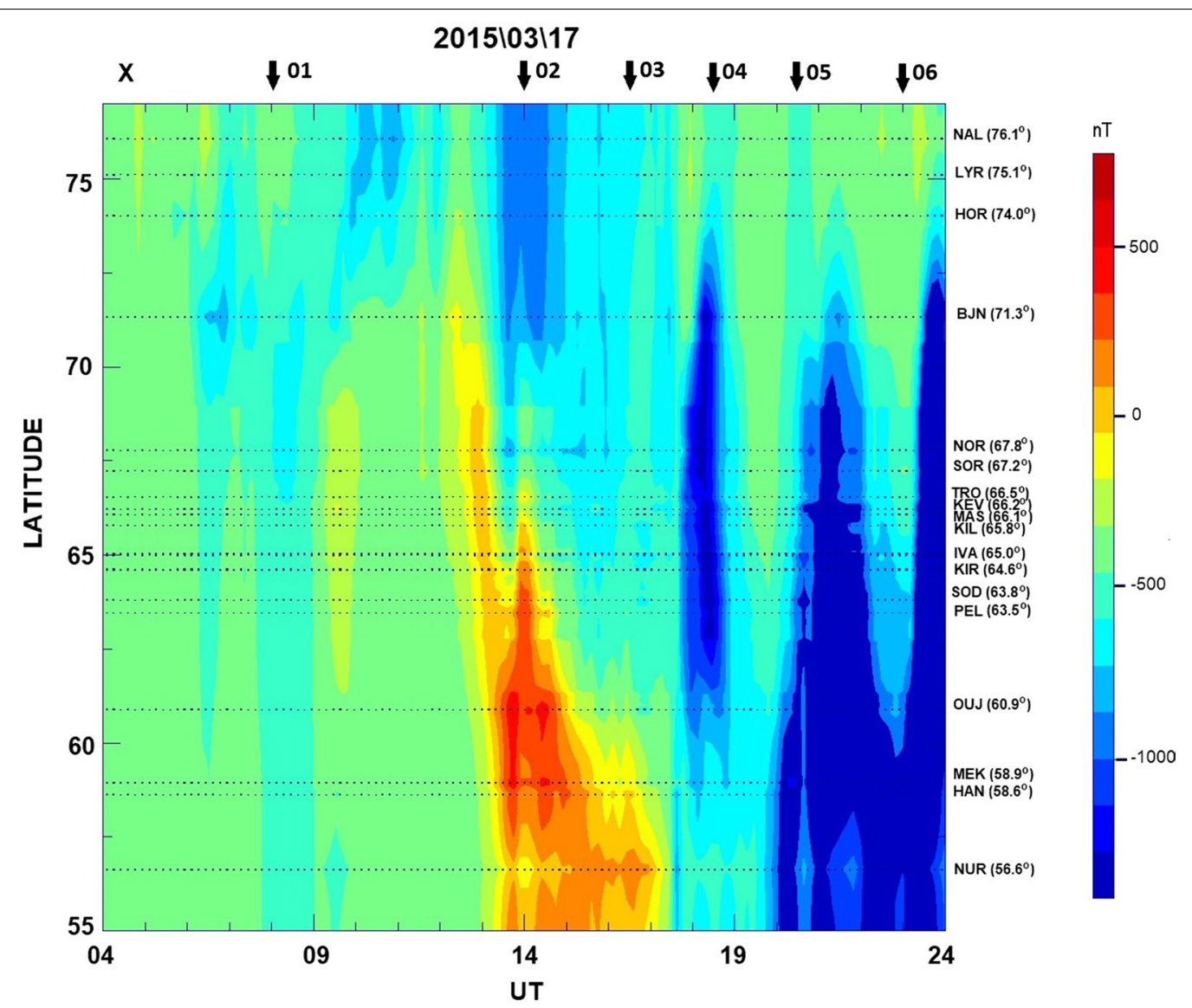

Fig. 6 Magnetic keogram showing the dynamics of latitudinal distribution of magnetic disturbance $\triangle X$ along the IMAGE profile during the storm main phase 04-24 UT. Blue color corresponds to the westward electrojet, yellow/red color corresponds to the eastward electrojet. Color coding is saturated to reveal signatures of weaker substorms. Near the right-hand $Y$-axis, the codes and geomagnetic latitudes of selected IMAGE stations are indicated. Arrows mark substorm \#1-6 activations

compared with subsequent substorms with comparable $\mathrm{AE}$ intensity. Bursts of GIC correspond well to bursts of magnetic field variability $|\mathrm{d} \mathbf{B} / \mathrm{d} t|$. The amplitudes of the $|\mathrm{d} \mathbf{B} / \mathrm{d} t|$ bursts at IMAGE stations near GIC sites, $\sim 100 \mathrm{nT} / \mathrm{min}$, are just somewhat less than effects recorded during the Quebec and Halloween superstorms, up to $300 \mathrm{nT} / \mathrm{min}$ (Wei et al. 2013). However, at some stations such as $\operatorname{BRW}\left(69.7^{\circ}, 250.1^{\circ}\right)$ and AND $\left(66.4^{\circ}, 100.9^{\circ}\right)$, the recorded $|\mathrm{d} \mathbf{B} / \mathrm{d} t|$ during the St. Patrick storm, $\sim 600 \mathrm{nT} / \mathrm{min}$, even supersedes the corresponding superstorm effects.

We compare the substorm intensity, as measured by the local IMAGE electrojet index IE (http://space.fmi.fi/ image/www/), the amplitude of the magnetic field variability $|\mathrm{dB} / \mathrm{d} t|$, and GIC peak intensity at IVA and VYH stations during all GIC bursts. This comparison shows that there is no linear relation between the substorm intensity and the level of geomagnetic variability. For example, substorm starting at $\sim 06$ UT with AE index $\sim 1500 \mathrm{nT}$ has a gradual growth and produces variations less $150 \mathrm{nT} / \mathrm{min}$. In contrast, substorms with similar peak intensity $\sim 1500-2000 \mathrm{nT}$ starting at $\sim 1730 \mathrm{UT}$ and $\sim 2340$ UT produce much larger variations, $\sim 700 \mathrm{nT} / \mathrm{min}$ and $\sim 360 \mathrm{nT} / \mathrm{min}$, respectively. Thus, AE/SME/IE indices are not a reliable measure of related GIC intensity.

\section{Discussion and conclusion}

We propose additional tools for the analysis of geomagnetic data from a world-wide array. These tools could be a useful augmentation of the existing online facilities. The FMI website (http://www.geo.fmi.fi/image ) provides ionospheric equivalent currents in the E-W direction, reconstructed from the $X$-components of the IMAGE latitudinal profile. Such plots are somewhat similar to our magnetic keogram ( $\Phi$-UT plots). The SuperMAG online facility (http://supermag.jhuapl.edu/ $\mathrm{mag} /$ ) gives a possibility to plot a snapshot of the $2 \mathrm{D}$ vector field of an instant magnetic disturbance. However, Ф-MLT plots with the power of geomagnetic disturbances are not available. None of the existing online 


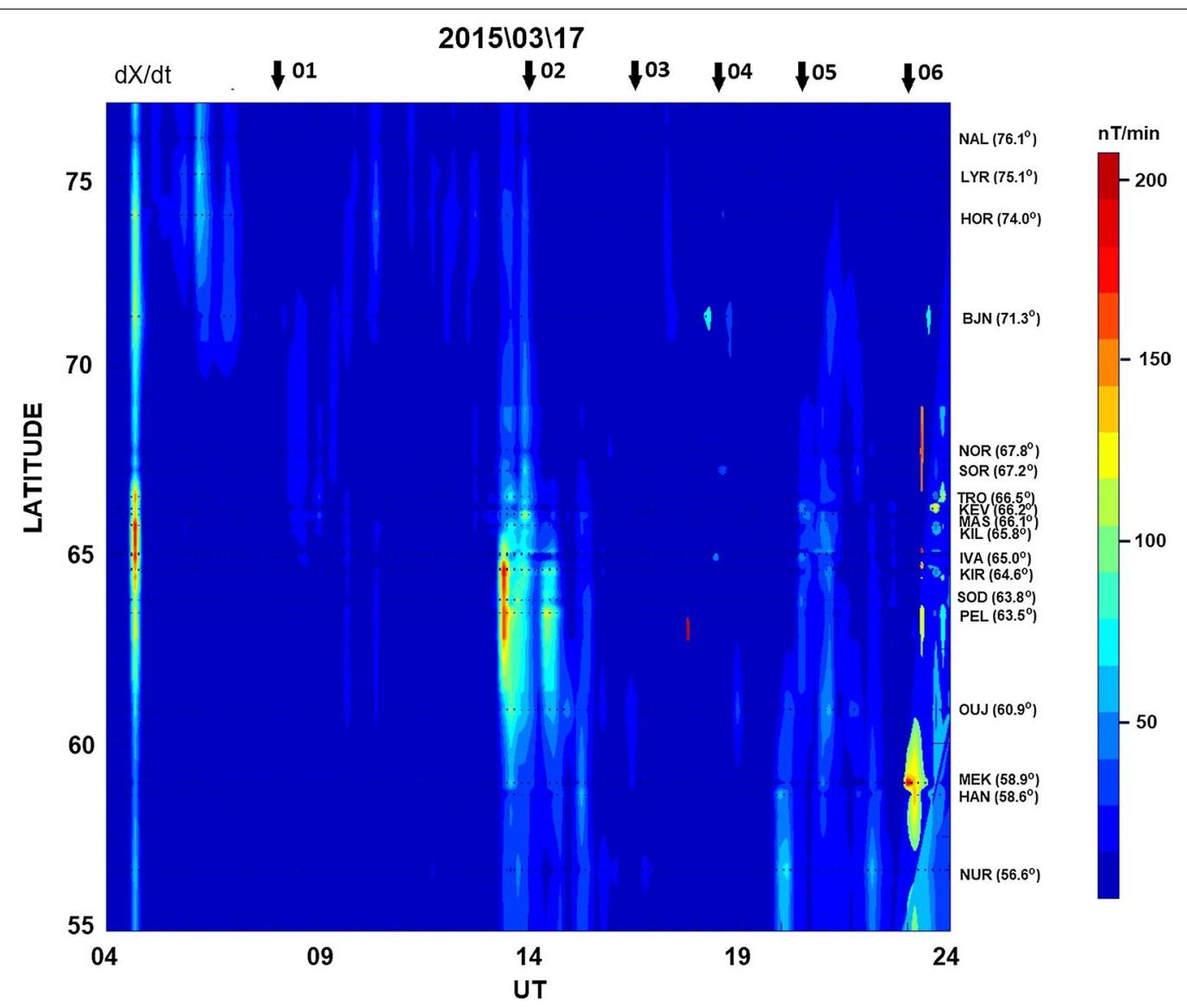

Fig. 7 Magnetic keogram showing the dynamics of latitudinal distribution of magnetic variability magnitude $|\mathrm{d} X / \mathrm{d} t|$ along the IMAGE profile during the storm main phase $04-24$ UT. Color coding is saturated to reveal signatures of weaker magnetic field variability. Codes and geomagnetic latitudes of selected IMAGE stations are indicated near the right-hand $Y$-axis. Arrows mark substorm \#1-6 activations

databases give information about the geomagnetic field variability $\mathrm{dB} / \mathrm{d} t$. Quick-look analysis of magnetic field response to the IMF/solar wind driving is greatly facilitated by the use of database with the pre-calculated VMs. These VMs where the spatial variations had been excluded are a convenient tool for quick-look browsing of such effects as day-night or N-S asymmetry.

We applied these tools for examination of fine features of the March 17, 2015, magnetic storm. Though the storm main phase is rather gradual $(\sim 17 \mathrm{~h}$ duration), it contains short-lived substorm-like activations with intensities up to $\sim 1500-2500 \mathrm{nT}$. The induction effect of these magnetic bursts was shown to cause bursts of GIC in electric power transmission lines on the Kola peninsula. However, no one-to-one correspondence between the substorm intensity, characterized by the local electrojet index, and the GIC intensity was found. One of the reasons may be related to a lack in the GIC vulnerability analysis of the Earth's geoelectrical structure beneath the power grid, and the topology and relative resistances of the power grid elements. This information is not available to us so far.

The application of techniques of the $\Phi$-MLT snapshots and magnetic keograms to magnetic variations $\Delta \mathbf{B}$ and time derivative $\mathrm{d} \mathbf{B} / \mathrm{d} t$ enabled us to reveal interesting features of the geomagnetic field variability, in particular, to indicate spatial location of fast variations responsible for GICs. The latitude-MLT mapping of magnetic disturbances from a world-wide array of magnetometers shows that the region with the highest magnetic variability does not coincide with a substorm "epicenter" but is predominantly shifted poleward. The magnetic keogram provides an easy-to-use visualization tool showing where the latitudinal structure of a region with elevated $|\mathrm{d} X / \mathrm{d} t|$ magnitude is. Significant GIC magnitudes would be observed only when a region with high variability, typically with scale $\Delta \Phi \sim 2^{\circ}-4^{\circ}$, overlaps with a power transmission line in question.

So far, we have used very simple and easy-to control gridding algorithms. The reasonable obtained results 


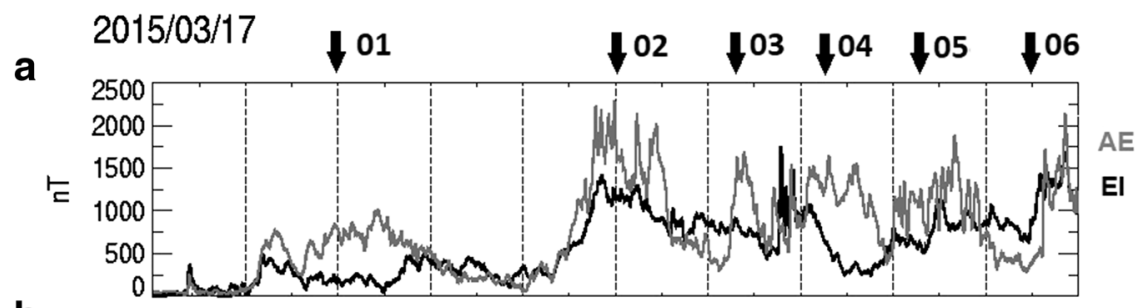

b
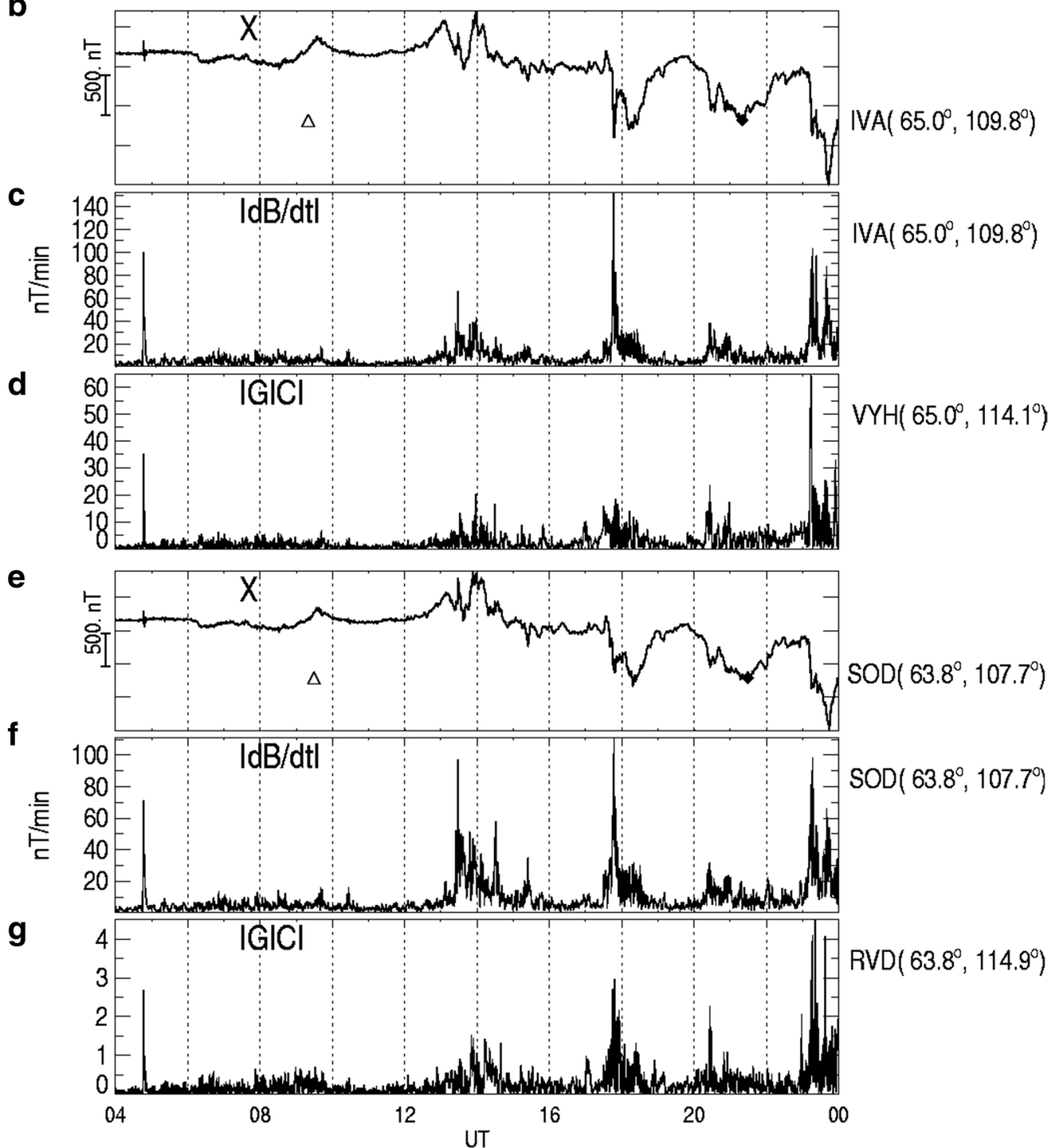

Fig. 8 Geomagnetic field variations and GICs in the power line system at stations VYH and RVD during March 17, 2015, 04-24 UT: a AE index (gray line) and local IMAGE El-index (black line); b magnetic field $\triangle X$ variations at IVA; $\mathbf{c}$ variability of geomagnetic field $|\mathrm{d} B / \mathrm{d} t|$ at IVA; $\mathbf{d}$ GIC magnitude at VYH; e magnetic field variations $\triangle X$ at SOD; $\mathbf{f}$ variability of geomagnetic field $|\mathrm{d} \mathbf{B} / \mathrm{d} t|$ at SOD; and $\mathbf{g} \mathrm{GIC}$ magnitude at RVD. Arrows mark substorm $\# 1-6$ activations

suggest the proposed technique, at least, has promise. Surely, at very large distances between stations the results become untrustworthy. Therefore, in the future we plan to test more advanced algorithms. However, even advanced algorithms cannot substitute the lack of stations in some regions.

The problem of GIC excitation during substorms is not just an "engineering" application of space physics 
research. It raises some fundamental questions. In particular, analysis of this event supports the view that the key factor of GIC excitation is the medium-scale and fastburst component of substorm evolution. This component becomes evident in variations of GIC intensity and magnetic field variability $|\mathrm{d} \mathbf{B} / \mathrm{d} t|$. Understanding the mechanisms of this burst process in auroral dynamics requires further studies and modeling.

\begin{abstract}
Abbreviations
AE: auroral electrojet; CIR: corotating interaction region; CME: coronal mass ejection; GIC: geomagnetically induced current; IMAGE: International Monitor for Auroral Geomagnetic Effects; IMF: interplanetary magnetic field; MLT: magnetic local time; N-S: north-south; OVATION: (Auroral) Oval Variation, Assessment, Tracking, Intensity, and Online Nowcasting; PC: polar cap; SME: SuperMag AE index; VM: virtual magnetogram.
\end{abstract}

\section{Authors' contributions}

OK processed the magnetometer data, VP wrote the article, VB processed the GIC data, and YS maintained the GIC stations. All authors read and approved the final manuscript.

\section{Author details \\ ${ }^{1}$ Institute of Physics of the Earth, Moscow, Russia. ${ }^{2}$ Geophysical Center, Mos- cow, Russia. ${ }^{3}$ Polar Geophysical Institute, Apatity, Russia.}

\section{Acknowledgements}

We appreciate thorough and in-depth examination of the manuscript by both Reviewers.

\section{Competing interests}

The authors declare that they have no competing interests.

\section{Availability of data and materials}

We acknowledge the provision of magnetometer data from SUPERMAG (http://supermag.jhuapl.edu), INTERMAGNET (http://www.intermagnet.org), CARISMA (http://www.carisma.ca), MACCS (http://space.augsburg.edu/maccs ), IMAGE (http://www.geo.fmi.f/image), Greenland Coastal (http://www.space .dtu.dk), GIMA (https://www.asf.alaska.edu/magnetometer), MAGDAS (http:// magdas.serc.kyushu-u.ac.jp), and Russian Arctic array (http://themis.ssl.berke ley.edu). Data of NASA OMNI-2 database (ftp://spdf.gsfc.nasa.gov/pub/data/ omni), AE/SYM indices (http://wdc.kugi.kyoto-u.ac.jp), PC index (http://pcind ex.org), and the OVATION model (http://sd-www.jhuapl.edu/Aurora/ovation/) are acknowledged.

\section{Funding}

This research was supported by the Russian Science Foundation Grant 16-17$00121(\mathrm{OK}, \mathrm{VP})$

\section{Publisher's Note}

Springer Nature remains neutral with regard to jurisdictional claims in published maps and institutional affiliations.

Received: 22 March 2018 Accepted: 25 September 2018

Published online: 01 October 2018

\section{References}

Boteler DH, Pirjola RJ, Nevanlinna H (1998) The effects of geomagnetic disturbances on electrical systems at the Earth's surface. Adv Space Res 22:17-27

Gjerloev JW (2012) The SuperMAG data processing technique. J Geophys Res 117:A09213. https://doi.org/10.1029/2012JA017683

Goldstein J, Angelopoulos V, DePascuale S et al (2017) Cross-scale observations of the 2015 St. Patrick's day storm: THEMIS, Van Allen Probes, and TWINS. J Geophys Res 122:368-392

Kamide Y, Kusano K (2015) No major solar flares but the largest geomagnetic storm in the present solar cycle. Space Weather 13:365-367

Kataoka R, Shiota D, Kilpua E, Keika K (2015) Pileup accident hypothesis of magnetic storm on 17 March 2015. Geophys Res Lett 42:5155-5161

Lanzerotti LJ (2001) Space weather effects on technologies. Space Weather 125:11

Le G, Lühr H, Anderson BJ et al (2016) Magnetopause erosion during the March 17, 2015, magnetic storm: combined field-aligned currents, auroral oval, and magnetopause observations. Geophys Res Lett. https://doi. org/10.1002/2016gl068257

LiW, LiW, Ma Q et al (2016) Radiation belt electron acceleration during the 17 March 2015 geomagnetic storm: observations and simulations. J Geophys Res 121:5520-5536

Lyons LR et al (2016) The 17 March 2013 storm: synergy of observations related to electric field modes and their ionospheric and magnetospheric effects. J Geophys Res 121:10880-10897

Newell PT, Gjerloev JW (2011) Evaluation of SuperMAG auroral electrojet indices as indicators of substorms and auroral power. J Geophys Res 116:A12211. https://doi.org/10.1029/2011JA016779

Ramsingh SS, Sreekumar S, Banola S, Emperumal K, Tiwari P, Kumar BS (2015) Low-latitude ionosphere response to super geomagnetic storm of 17/18 March 2015: results from a chain of ground-based observations over Indian sector. J. Geophys. Res. Space Physics 120:10864-10882

Sakharov YA, Danilin AN, Ostafiychuk RM, Katkalov YV, Kudryashova NV (2009) Geomagnetically induced currents in the power systems of the Kola peninsula at solar minimum. In: Proceedings of 8th international symposium on electromagnetic compatibility and ecology, St-Ptb, pp 237-238

Stauning P (2018) Multi-station basis for Polar Cap (PC) indices: ensuring credibility and operational reliability. I Space Weather Space Climate 8:A07. https://doi.org/10.1051/swsc/2017036

Viljanen A (1997) The relation between geomagnetic variations and their time derivatives and implications for estimation of induction risks. Geophys Res Lett 24:631-634

Viljanen A (2011) European project to improve models of geomagnetically induced currents. Space Weather 9:507007

Viljanen A, Nevanlinna H, Pajunpaa K, Pulkkinen A (2001) Time derivative of the horizontal geomagnetic field as an activity indicator. Ann Geophys 19:1107-1118

Wei LH, Homeier N, Gannon JL (2013) Surface electric fields for North America during historical geomagnetic storms. Space Weather 11:451-462

\section{Submit your manuscript to a SpringerOpen ${ }^{\circ}$ journal and benefit from:}

- Convenient online submission

- Rigorous peer review

- Open access: articles freely available online

- High visibility within the field

- Retaining the copyright to your article

Submit your next manuscript at $\boldsymbol{\nabla}$ springeropen.com 